\title{
El futuro trascendental como determinante de las conductas sustentables y la percepción de felicidad
}

\section{Transcendental future as a determinant of sustainable behavior and the perception of happiness}

\author{
Laura Fernanda Barrera-Hernández 1 ORCID , Víctor Corral-Verdugo 2 ORCID, \\ Blanca Silvia Fraijo-Sing 3 oRCID \\ ${ }^{1}$ Instituto Tecnológico de Sonora \\ 23 Universidad de Sonora \\ México
}

Fecha correspondencia:

Recibido: septiembre 17 de 2019.

Aceptado: diciembre 15 de 2020.

\section{Forma de citar:}

Barrera-Hernández, L.F., Corral-

Verdugo, V., \& Fraijo-Sing, B.S.

(2021). El futuro trascendental como

determinante de las conductas

sustentables y la percepción de

Felicidad. Rev. CES Psico, 14(1), 1-15.

\section{Open access}

(c) Copyright

Licencia creative commons

Ética de publicaciones

Revisión por pares

Gestión por Open Journal System

DOI: http://dx.doi.org/10.21615/

cesp.14.1.2

ISSN: 2011-3080

Comparte

\section{Resumen}

La perspectiva temporal es una variable psicológica que se ha asociado con múltiples comportamientos, entre ellos la conducta sustentable (CS). Son escasos los estudios sobre la relación entre la orientación temporal denominada futuro trascendental y la CS. Objetivo: estimar las relaciones entre dos perspectivas temporales (orientación al futuro y orientación al futuro trascendental) y la conducta sustentable; y su asociación, con el autocuidado y la felicidad. Metodología: estudio correlacional, con un diseño no experimental, transeccional. Participaron 209 personas de una ciudad al noroeste de México, con una edad promedio de 30.33 años, quienes respondieron a un instrumento que constó de ocho escalas Likert que evaluaron perspectiva de futuro, perspectiva temporal de futuro trascendental, conducta proecológica, altruismo, frugalidad, equidad, comportamientos de autocuidado y felicidad subjetiva. Las interrelaciones entre las variables se estimaron mediante un modelo de ecuaciones estructurales. Resultados: las orientaciones temporales al futuro y al futuro trascendental influyen en las conductas sustentables (conducta proecológica, altruismo, frugalidad, equidad), que, a su vez, impactan en la felicidad y el autocuidado de las personas. Conclusiones: Estos hallazgos aportan información valiosa que ayuda a elucidar las características que posee un individuo que orienta su conducta a los ideales de la sostenibilidad.

Palabras clave: Orientación al Futuro, Futuro Trascendente, Conducta Sustentable, Conducta Proecológica, Felicidad, Autocuidado.

\section{Abstract}

Time perspective is a psychological variable that has been associated it with multiple behaviors, including sustainable behaviors. Research on transcendental future perspective is scarce, among the studies on temporal perspective and sustainable behavior, none were found on the relationship between transcendental future and SC. Objective: this research was aimed at assessing the relationship between two-time perspectives 


\section{Sobre los autores:}

1. Doctora en Ciencias

Sociales. Profesora-

investigadora del

departamento de Psicología

en el Instituto Tecnológico de

Sonora.

2. Doctor en Psicología

Ambiental. Profesor titular

de psicología en la División

de Ciencias Sociales de la

Universidad de Sonora.

3. Doctora en Ciencias

Sociales. Profesora-

investigadora del

Departamento de Psicología y

Ciencias de la Comunicación

de la Universidad de Sonora. (future orientation, transcendent future orientation) and sustainable behavior, and its association with self-care and happiness. Method: a correlational study was developed, with a non-experimental, transactional design. Two hundred nine people from a northwestern city in Mexico participated, with an average age of 30.33 years, who responded to an instrument that consisted of eight Likert scales that evaluated future perspective, transcendental future temporal perspective, pro-ecological behavior, altruism, frugality, equity, self-care behaviors, and subjective happiness.

Results: the results were processed within a structural equation model, where it is emphasized that temporal orientations to the future and to the transcendental future influence sustainable behaviors (pro-ecological behavior, altruism, frugality, equity), which, in turn, impact on the happiness and self-care of people. Conclusion: these findings provide valuable information that helps elucidate the characteristics of an individual who guides his behavior to sustainability ideals.

Keywords: Future Orientation, Transcendent Future, Sustainable Behavior, Pro-Ecological Behavior, Happiness, Self Care.

\section{Introducción}

En la experiencia humana, la percepción del tiempo es una característica fundamental que puede asumir una vertiente objetiva, considerada como tiempo de reloj, o subjetiva, a través de las construcciones personales del tiempo (Boniwell \& Zimbardo, 2004). La perspectiva temporal (TP) es un proceso mediante el cual se asignan categorías temporales a las experiencias personales y sociales para dar orden, coherencia y sentido a los eventos (Zimbardo \& Boyd, 1999). Corral (2010) indica que para la mayoría de las personas el tiempo es fundamental debido a que son conscientes de su finitud, por lo cual realizan cálculos para planear sus vidas.

Zimbardo y Boyd (1999) plantearon un modelo de perspectiva temporal que incluye cinco factores: pasado negativo, pasado positivo, presente fatalista, presente hedonista y futuro, los cuales puedes ser medidos a través del Zimbardo Time Perspective Inventory (ZTPI). El primer factor, el pasado negativo refleja una visión generalmente negativa o aversiva del pasado, que puede derivarse de experiencias reales de acontecimientos desagradables o traumáticos, de la reconstrucción negativa de eventos benignos o de una mezcla o de ambos. El pasado positivo se refiere a una actitud cálida y sentimental hacia el pasado. Por su parte, el presente fatalista revela una actitud fatídica, de indefensión y desesperanza hacia el futuro y la vida. El presente hedonista refleja una actitud centrada en el placer hacia la vida aquí y ahora, toma de riesgos y poca preocupación por las consecuencias futuras. Finalmente, la orientación hacia el futuro sugiere que el comportamiento está dominado por una aspiración a metas y recompensas en el tiempo por venir.

Estos autores también propusieron la perspectiva temporal de futuro trascendental, que divide el futuro en tramas anteriores y posteriores a la muerte; esta orientación temporal se extiende desde la proximidad a la muerte imaginada del cuerpo físico hasta el infinito. Entre las metas de las personas que se orientan por esta perspectiva están: reunión con seres queridos que han fallecido, inicio de la vida eterna, evasión de la condenación eterna, desaparición del dolor, el sufrimiento y la vergüenza (Boyd \& Zimbardo, 1997). 
Barrera-Hernández, Corral-Verdugo, Fraijo-Sing y Tapia-Fonllem (2018) encontraron una asociación significativa entre un entorno trascendental (escenario que resulta de la relación entre las personas y sus ideas respecto a entidades y procesos trascendentales) y el comportamiento sustentable, indicando que cuanto más inmersa está una persona en un ambiente trascendental positivo, realizará más comportamientos proecológicos, altruistas, equitativos y frugales (estos últimos hacen alusión a la reducción o evitación del consumo de recursos).

\section{Perspectiva temporal de futuro trascendental}

La perspectiva temporal de futuro trascendental predice la recompensa eterna o el castigo en la otra vida, lo cual es de suma importancia para muchos individuos y puede afectar sustancialmente su vida presente (Van Beek \& Kairys, 2015).

Zimbardo y Boyd (1999) señalan que los individuos que puntúan alto en la Escala de perspectiva de tiempo futuro trascendental (TFTP, por sus siglas en inglés de Trascendental Future Time Perspective) (Boyd \& Zimbardo, 1997), que evalúa la orientación al futuro trascendental, creen que van a ser premiados o castigados por su comportamiento presente, al igual que lo hacen las personas que obtienen una puntuación alta en la subescala tradicional de futuro, pero con la diferencia de que para aquellos con orientación al futuro trascendental el refuerzo viene sólo después de su muerte. Desde esta perspectiva temporal, algunos comportamientos considerados a menudo irracionales como el suicidio, el heroísmo extremo y la entrega excesiva de diezmos se transforman en conductas racionales previstas para llevar al cumplimiento de objetivos trascendentales futuros (Boyd \& Zimbardo, 1997).

Boyd y Zimbardo (1997) hallaron que el modelo de persona orientada al futuro trascendental se caracteriza por: ser de edad avanzada, mujer, protestante, de origen hispano o afroamericano; además, por valorar la salvación, asistir a templos, orar y efectuar rituales en casa. Ortuño, Paixão y Nunes (2011) desarrollaron un estudio con estudiantes universitarios que respondieron a la escala TFTP, y encontraron que los individuos practicantes de una religión puntuaron mucho más alto en la escala de orientación al futuro trascendental que quienes no lo eran. Diversos estudios (Ortuño, Paixão, \& Nunes, 2013; Seema, Baltin, \& Sircova, 2014), encontraron una diferencia estadísticamente significativa en los resultados de la escala TFTP según el sexo, siendo las mujeres quienes presentaron mayor orientación al futuro trascendental respecto a los hombres. Ortuño, Paixão y Nunes (2013), además, reportaron correlaciones significativas entre la escala TFTP y los otros marcos temporales de la escala ZTPI. Barrera-Hernández, Corral-Verdugo, Fraijo-Sing y Tapia-Fonllem (2018) encontraron una asociación significativa entre un entorno trascendental (escenario que resulta de la relación entre las personas y sus ideas respecto a entidades y procesos trascendentales) y el comportamiento sustentable, indicando que cuanto más inmersa está una persona en un ambiente trascendental positivo, realizará más comportamientos proecológicos, altruistas, equitativos y frugales (estos últimos hacen alusión a la reducción o evitación del consumo de recursos).

Lee (2009) indica que la investigación acerca de la perspectiva temporal de futuro generalmente se centra en las orientaciones futuras de los individuos con respecto a su espacio de vida personal, esto es, con experiencias ligadas a la vida y no a la muerte. Van Beek y Kairys (2015) señalaron que la investigación acerca de la perspectiva temporal de futuro trascendental es verdaderamente escasa.

\section{Conducta Sustentable}

Las conductas sustentables (CS) son el conjunto de acciones que se encaminan a la conservación de los recursos naturales y sociales; lo que supone garantizar la integridad de las especies animales y vegetales existentes, así como el bienestar individual y social de las generaciones actuales y futuras (Corral \& Pinheiro, 2004). Esta definición considera el componente de temporalidad de manera evidente y explícita, declarando que uno de sus objetivos centrales es garantizar la calidad ambiental para el futuro. 
Arnocky, Milfont y Nicol (2014) indican que la decisión de comportarse de manera sustentable requiere de un enfoque en los resultados futuros a expensas de los beneficios inmediatos, debido a que los beneficios a largo plazo del comportamiento ambiental a menudo implican costos directos en el presente; por ejemplo, el tiempo y el dinero gastados en el corto plazo.
Corral (2010) incluye entre las CS los comportamientos proecológicos, entendidos como el conjunto de acciones intencionales y efectivas que resultan en la conservación del ambiente natural; las conductas frugales o de austeridad, que reflejan un estilo de vida que promueve la reducción o evitación del consumo de recursos; las conductas altruistas que involucran acciones cuyo objetivo es incrementar el bienestar de otras personas; y las conductas equitativas, vinculadas con la premisa de que los seres humanos son en esencia iguales, independientemente de la diversidad de rasgos que los caracterizan.

\section{La orientación al futuro como antecedente de la conducta sustentable}

La perspectiva temporal se ha considerado como un factor disposicional o predictivo de las CS. Arnocky, Milfont y Nicol (2014) indican que la decisión de comportarse de manera sustentable requiere de un enfoque en los resultados futuros a expensas de los beneficios inmediatos, debido a que los beneficios a largo plazo del comportamiento ambiental a menudo implican costos directos en el presente; por ejemplo, el tiempo y el dinero gastados en el corto plazo. Ante esta situación los investigadores han prestado cada vez más atención al rol que juega la perspectiva temporal en las decisiones de los individuos para comprometerse en pro del medio ambiente.

Entre los estudios que abordan la relación entre las CS y la perspectiva temporal se encuentra el realizado por Corral-Verdugo, Fraijo-Sing y Pinheiro (2006), en el que encontraron que la orientación al futuro se relacionaba positivamente con el ahorro de agua. Asimismo, Milfont y Gouveia (2006) hallaron que la preservación del medio ambiente se relaciona positivamente con una orientación al futuro. Fraijo, Corral, Tapia y García (2012) reportaron que la propensión al futuro se correlaciona de manera positiva y significativa con la deliberación o intención proambiental, la austeridad, la equidad, el altruismo y el comportamiento proecológico. Finalmente, Enzler (2015) señaló que la consideración de consecuencias futuras es un predictor significativo del comportamiento proambiental.

\section{CS y Felicidad}

Las CS también son instigadas por consecuencias positivas que resultan de su ejecución (Corral, 2010); al respecto, diversas investigaciones (Corral-Verdugo, Mireles-Acosta, Tapia-Fonllem \& Fraijo-Sing, 2011; Madjar \& Ozawa, 2006; Manríquez-Betanzos, Corral-Verdugo, Vanegas-Rico, Fraijo-Sing, \& Tapia-Fonllem, 2016; Tapia-Fonllem, Corral-Verdugo, Fraijo-Sing \& Durón-Ramos, 2013) han demostrado que existe una relación significativa entre las CS y la felicidad.

Brown y Kasser (2005) mencionan que la felicidad y el bienestar ecológico pueden ser complementarios. En este sentido, Corral (2012) señala que las prácticas prosociales y proambientales pueden ayudar a incrementar los niveles de bienestar subjetivo.

Por su parte, Brown y Kasser (2005) reportan una correlación significativa entre el bienestar subjetivo y el comportamiento ecológicamente responsable; además, una asociación positiva de la generosidad (o altruismo, un indicador de las CS) con el bie- nestar subjetivo y el comportamiento ecológicamente responsable. Corral-Verdugo et al. (2011) encontraron que las CS influyen significativamente en la felicidad; de modo que entre más proecológica, altruista, frugal y equitativa sea una persona, mayor es el sentimiento de felicidad que experimenta. Csutora y Zsóka (2012) señalaron que las personas más felices actúan de manera más amigable con el medio 
La presente investigación tiene como propósito estimar las relaciones entre dos perspectivas temporales (orientación al futuro y orientación al futuro trascendental) y las CS; y las asociaciones entre dichas perspectivas temporales, con el autocuidado y la felicidad. Se parte de la hipótesis de que la orientación al futuro y al futuro trascendental influirían en la práctica de conductas sustentables y que éstas resultarían en niveles más elevados de felicidad, promoviendo, asimismo, conductas de autocuidado en quienes las practican. ambiente. Choi (2016) reveló correlaciones positivas y estadísticamente significativas entre comportamiento proecológico, equidad, altruismo y felicidad. Asimismo, Barrera-Hernández, Sotelo-Castillo, Echeverría-Castro y Tapia-Fonllem (2020) reportaron una asociación positiva entre las CS y la felicidad.

Landes, Unger, Andsbjerg, Frank y Wiking (2015) indicaron que la investigación acerca de la relación entre los comportamientos sustentables y la felicidad aún es incipiente.

\section{Autocuidado y CS}

Las conductas de autocuidado, según Tobón (2003), se relacionan con aquellos cuidados que la persona se proporciona para lograr una mejor calidad de vida. Pato y Corral (2013) corroboraron la hipótesis de que las personas que practican conductas de autocuidado también practican comportamientos de cuidado de otras personas (conductas prosociales) y del medio ambiente natural. Dada la relación entre la conducta de autocuidado y la CS, se incluyó esta variable al modelo de relaciones que se especificó en el presente estudio.

Considerando lo anterior, la presente investigación tiene como propósito estimar las relaciones entre dos perspectivas temporales (orientación al futuro y orientación al futuro trascendental) y las CS; y las asociaciones entre dichas perspectivas temporales, con el autocuidado y la felicidad. Se parte de la hipótesis de que la orientación al futuro y al futuro trascendental influirían en la práctica de conductas sustentables y que éstas resultarían en niveles más elevados de felicidad, promoviendo, asimismo, conductas de autocuidado en quienes las practican.

\section{Método}

Estudio correlacional, con un diseño no experimental, transeccional (Kerlinger \& Lee, 2002).

\section{Participantes}

Mediante un muestreo no probabilístico, se conformó una muestra integrada por 209 personas de la ciudad de Hermosillo, Sonora (México), $56.5 \%$ mujeres y $41.1 \%$ hombres (2.4\% no reportó el sexo), con edades comprendidas entre los 16 y los 82 años, con un promedio de 30.33 años ( $D E=13.88$ ). El $64.4 \%$ de los participantes era soltero, $22.5 \%$ casado, $5.3 \%$ vivía en unión libre; $3.8 \%$ reportó ser viudo y 3.3\% divorciado. Respecto al nivel de escolaridad, $70.8 \%$ poseía estudios universitarios, $11.5 \%$ de bachillerato, $7.7 \%$ de secundaria, $6.2 \%$ de posgrado y el resto de educación primaria.

\section{Instrumentos}

Escala de Futuro del Inventario de Perspectiva Temporal de Zimbardo (ZTPI) (Zimbardo \& Boyd, 1999). Adaptada a población mexicana (Corral-Verdugo et al., 2006), mide situaciones y creencias características de personas con orientación al futuro, tales como planear los días, proponerse metas y cumplir a tiempo con obligaciones. La escala consta de 12 ítems en escala Likert con cinco opciones de respuesta (1= muy poco aplicable hasta 5 =bastante aplicable). En este estudio la confiabilidad de la escala fue de $\alpha=.83$.

Escala de Futuro Trascendental (TFTP) (Zimbardo \& Boyd, 2008). Adaptada por Corral (2010), consta de 10 ítems en escala Likert que reflejan creencias en la vida después de la muerte y la rendición de cuentas por las acciones realizadas en vida, tales como la muerte como un nuevo comienzo, los humanos poseen alma, etcétera. La 
escala contiene cinco opciones de respuesta ( $1=$ nada verdadero hasta $5=$ muy ver dadero). En este estudio la confiabilidad de la escala fue de $a=.88$.

Escala de Conducta Proecológica General de Kaiser (1998). La versión adaptada a población mexicana por Tapia et al. (2006) consta de 16 ítems en escala Likert, en los que los participantes autoreportan la frecuencia de comportamientos dirigidos al cuidado del ambiente natural (reutilización, reciclaje, conservación de energía, etc.); mediante cuatro opciones de respuesta que van de $0=$ nunca a $3=$ siempre. La confiabilidad de la escala en este estudio fue $\alpha=.71$.

Escala de Altruismo de Corral-Verdugo et al. (2010). Conformada por 10 reactivos en escala tipo Likert que describen conductas de ayuda desinteresada a otras personas, tales como donar sangre, visitar enfermos, ayudar económicamente a los pobres, entre otras; con cuatro opciones de respuesta que van de $0=$ nunca a $3=$ siempre. En esta investigación el índice de consistencia interna de la escala fue $a=.79$.

Escala de Frugalidad (Corral \& Pinheiro, 2004). Consta de diez ítems en escala Likert, que enuncian conductas de vida austera, vivir sin lujos, reusar ropa y comprar lo estrictamente necesario. La escala cuenta con cinco opciones de respuesta que van de $0=$ totalmente en desacuerdo a $4=$ totalmente de acuerdo. La confiabilidad de la escala en la presente investigación fue de $\alpha=.77$.

Escala de Equidad (Corral-Verdugo et al., 2010). Contiene siete ítems en escala Likert que refieren al trato igualitario e igualdad de derechos y obligaciones, entre sexos, diferentes edades, condiciones socioeconómicas y razas. Los participantes determinan su grado de acuerdo, empleando una escala de respuesta que va del $0=$ totalmente en desacuerdo a $4=$ totalmente de acuerdo. La confiabilidad de la escala en esta investigación fue $\alpha=.71$.

Escala de Autocuidado (Corral \& Pato, 2013). Mide conductas de cuidado consigo mismo como practicar actividad física regularmente, meditar, alimentarse sanamente, entre otras. La escala está conformada por 18 ítems en escala Likert con cinco opciones de respuesta $(0=$ nunca a $4=$ todos los días). La confiabilidad de la escala en este estudio fue de $a=.86$.

Escala de Felicidad Subjetiva (SHS, por sus siglas de Subjective Happiness Scale) (Lyubomirsky \& Lepper, 1999). La versión adaptada a población mexicana por Corral-Verdugo et al. (2011) constituye una medida de la felicidad global y requiere que los participantes indiquen su percepción de felicidad experimentada por medio de ítems tales como "En general me considero feliz" y "Comparado con la mayoría de mis compañeros, me considero feliz", en un rango de respuestas que va del $1=$ no muy feliz al 7= muy feliz. La confiabilidad de la escala en este estudio fue de $\alpha=.87$.

\section{Procedimiento}

La recolección de datos fue realizada por un grupo de encuestadores previamente capacitados. La aplicación del instrumento (reúne las diversas escalas) se llevó a cabo en las viviendas de los participantes y áreas recreativas, tales como parques y plazas públicas ubicados en la zona centro de la ciudad. A los potenciales participantes se les invitó a colaborar y se les explicó el objetivo del estudio, una vez que aceptaron participar de manera voluntaria, se les entregó un formato de consentimiento informado, que especificaba la confidencialidad de los datos recabados y su uso exclusivo para fines de investigación; posteriormente, se proporcionó la versión 
En la variable de Comportamiento Proecológico los participantes presentaron una puntuación media de 1.52 y en Altruismo de 1.55 , esto en escalas que van del 0 al 3, lo que indica una frecuencia ocasional de conductas proecológicas o altruistas. de lápiz y papel del instrumento auto aplicable, y el tiempo de respuesta aproximado fue de 10 minutos. Los datos recolectados han sido protegidos atendiendo los lineamientos establecidos en la Declaración de Helsinki.

\section{Análisis de datos}

Los datos fueron analizados utilizando estadísticas univariadas (medias, desviación estándar y frecuencias) y se revisó la consistencia interna de las escalas calculando el alfa de Cronbach para cada una de ellas en SPSS versión 21.0.

Las interrelaciones entre las variables se estimaron mediante un modelo de ecuaciones estructurales en el paquete estadístico EQS 6.1, usando parcelas para los constructos estudiados, y los tres ítems de la Escala de Felicidad. Se construyeron ocho factores de primer orden: 1) Futuro, 2) Futuro Trascendental, 3) Felicidad, 4) Autocuidado, 5) Frugalidad, 6) Comportamiento Proecológico, 7) Altruismo y 8) Equidad; y un factor de segundo orden denominado "conducta sustentable" conformado a partir de los factores Frugalidad, Comportamiento Proecológico, Altruismo y Equidad.

El modelo especificado hipotetiza que los factores de orientación temporal al futuro y futuro trascendental influirían en el factor de segundo orden "conducta sustentable" a la vez que éste influiría significativamente en la felicidad y en las conductas de autocuidado.

\section{Resultados}

En la variable de Comportamiento Proecológico los participantes presentaron una puntuación media de 1.52 y en Altruismo de 1.55, esto en escalas que van del 0 al 3 , lo que indica una frecuencia ocasional de conductas proecológicas o altruistas. Por otra parte, en la variable Frugalidad obtuvieron una media de 2.37, en Autocuidado 2.42 y en Equidad de 3.50 (estas escalas van de 0 a 4), lo que indicaría niveles medios de Frugalidad y Autocuidado, y un alto nivel de Equidad; es decir, que los participantes refieren estar en parte de acuerdo con llevar acciones frugales y de autocuidado, y totalmente de acuerdo con la mayoría de las ideas sobre equidad. Respecto a la perspectiva temporal, se halló una media de 3.71 en la orientación al Futuro y 3.49 en la orientación al Futuro Trascendental (en una escala de 1 a 5); lo que indica que los participantes se encuentran altamente orientados hacia ambas perspectivas. Finalmente, en la variable Felicidad los participantes obtuvieron una puntuación media de 5.34 en una escala de 1 a 7, lo que indica un nivel alto de felicidad. En la Tabla 1 se presentan los resultados de los análisis estadísticos descriptivos y la consistencia interna de las escalas utilizadas. 
Tabla 1. Estadísticos descriptivos y confiabilidad de las escalas utilizadas

\begin{tabular}{|c|c|c|c|c|c|}
\hline Escalas / Ítems & Min & Max & $M$ & $D E$ & Alfa \\
\hline FUTURO & 1.33 & 5 & 3.71 & 0.64 & 0.83 \\
\hline Me incomoda llegar tarde a mis compromisos & 1 & 5 & 4.07 & 0.94 & \\
\hline Cumplo a tiempo mis obligaciones con mis amigos y autoridades & 2 & 5 & 4.01 & 0.87 & \\
\hline $\begin{array}{l}\text { Cumplir con los plazos que están por vencerse y hacer las cosas necesarias son cosas que vienen } \\
\text { primero que la diversión }\end{array}$ & 1 & 5 & 3.95 & 0.91 & \\
\hline Me preocupo si las cosas no se hacen a tiempo & 1 & 5 & 3.86 & 0.95 & \\
\hline Antes de tomar una decisión, evalúo costos y beneficios de esa decisión & 1 & 5 & 3.75 & 1.13 & \\
\hline $\begin{array}{l}\text { Cuando quiero conseguir algunas cosas, me propongo metas y evalúo los recursos con que } \\
\text { cuento, para alcanzar esos objetivos }\end{array}$ & 1 & 5 & 3.73 & 1.14 & \\
\hline Sigo trabajando en tareas difíciles y no interesantes, si ellas me van a ayudar a avanzar & 1 & 5 & 3.69 & 1.07 & \\
\hline Soy capaz de resistir las tentaciones cuando sé que hay trabajo por hacer & 1 & 5 & 3.67 & 1.1 & \\
\hline $\begin{array}{l}\text { Termino mis proyectos a tiempo porque mantengo un constante avance de actividades de ese } \\
\text { proyecto }\end{array}$ & 1 & 5 & 3.63 & 1.08 & \\
\hline Siempre va a haber tiempo para poner al día mi trabajo & 1 & 5 & 3.57 & 1.13 & \\
\hline Pienso que las personas deberían planear su día cada mañana & 1 & 5 & 3.42 & 1.04 & \\
\hline Hago listas de las cosas que tengo que hacer & 1 & 5 & 3.18 & 1.31 & \\
\hline FUTURO TRANSCENDENTAL & 1.2 & 5 & 3.49 & 0.92 & 0.88 \\
\hline Los humanos poseen un alma & 1 & 5 & 3.87 & 1.21 & \\
\hline Las leyes científicas no pueden explicar todo & 1 & 5 & 3.68 & 1.28 & \\
\hline Sólo mi cuerpo físico morirá & 1 & 5 & 3.63 & 1.25 & \\
\hline La teoría de la evolución explica adecuadamente cómo los humanos llegamos a ser lo que somos & 1 & 5 & 3.63 & 1.08 & \\
\hline Creo en los milagros & 1 & 5 & 3.5 & 1.4 & \\
\hline Creo en los espíritus & 1 & 5 & 3.43 & 1.37 & \\
\hline La muerte es sólo un nuevo comienzo & 1 & 5 & 3.38 & 1.35 & \\
\hline Mi cuerpo es sólo un hogar temporal para mi real Yo & 1 & 5 & 3.35 & 1.37 & \\
\hline Seré llamado a rendir cuentas por mis acciones en la tierra cuando muera & 1 & 5 & 3.33 & 1.37 & \\
\hline Hay leyes divinas que debieran regir las vidas de los seres humanos & 1 & 5 & 3.17 & 1.44 & \\
\hline CONDUCTA PROECOLÓGICA & 0.56 & 2.88 & 1.52 & 0.43 & 0.71 \\
\hline Espero tener una carga completa de ropa antes de meterla a la lavadora & 0 & 3 & 2.21 & 0.937 & \\
\hline Utilizo la secadora de ropa & 0 & 3 & 2.16 & 1.1 & \\
\hline Mato insectos con un insecticida químico & 0 & 3 & 1.91 & 1.01 & \\
\hline Compro productos (frutas y verduras) de temporada & 0 & 3 & 1.87 & 0.94 & \\
\hline Manejo en las vías rápidas a velocidades menores a 60 kph & 0 & 3 & 1.81 & 1.15 & \\
\hline Busco manera de reusar cosas & 0 & 3 & 1.8 & 0.91 & \\
\hline Compro comidas preparadas & 0 & 3 & 1.61 & 0.87 & \\
\hline Compro productos en empaques que pueden volver a utilizarse & 0 & 3 & 1.56 & 0.84 & \\
\hline Ahorro gasolina, caminando o viajando en bicicleta & 0 & 3 & 1.55 & 1.15 & \\
\hline Guardo y reciclo el papel usado & 0 & 3 & 1.39 & 1.14 & \\
\hline Le he hecho saber a alguien que se ha comportado de manera que dañe el ambiente & 0 & 3 & 1.34 & 1.03 & \\
\hline Leo acerca de temas ambientales & 0 & 3 & 1.21 & 0.97 & \\
\hline Platico con amigos acerca de problemas relacionados con el ambiente & 0 & 3 & 1.15 & 0.94 & \\
\hline Animo a mis amigos y familiares para que reciclen & 0 & 3 & 1.14 & 1.04 & \\
\hline Separo botellas vacías para reciclar & 0 & 3 & 1.06 & 1.11 & \\
\hline En el verano apago el aire acondicionado o cooler cuando dejo mi casa por más de cuatro horas & 0 & 3 & 0.63 & 0.95 & \\
\hline
\end{tabular}


Pág 9

\begin{tabular}{|c|c|c|c|c|c|}
\hline Escalas / Ítems & Min & Max & M & $D E$ & Alfa \\
\hline ALTRUISMO & 0 & 3 & 1.55 & 0.58 & 0.79 \\
\hline $\begin{array}{l}\text { Colaborar con sus compañeros de escuela o del trabajo a explicarles y ayudarles en tareas que no } \\
\text { entienden }\end{array}$ & 0 & 3 & 2.06 & 0.98 & \\
\hline Regalar ropa usada que ya no utiliza pero que está en buen estado. & 0 & 3 & 2.05 & 0.95 & \\
\hline Brindar atención a alguna persona que tropieza, o que se cae, o que se lastima en la calle & 0 & 3 & 2.01 & 0.96 & \\
\hline Guiar a personas para localizar alguna dirección. & 0 & 3 & 1.97 & 0.93 & \\
\hline Contribuir económicamente con la Cruz Roja. & 0 & 3 & 1.75 & 1 & \\
\hline Regalar una moneda a indigentes (pobres en la calle). & 0 & 3 & 1.75 & 0.92 & \\
\hline Ayudar a personas mayores o incapacitados a cruzar la calle. & 0 & 3 & 1.48 & 1.05 & \\
\hline $\begin{array}{l}\text { Participar en eventos para recolectar fondos para organizaciones civiles como los bomberos, la } \\
\text { Cruz Roja, etc. }\end{array}$ & 0 & 3 & 1 & 1 & \\
\hline Visitar a enfermos en hospitales. & 0 & 3 & 0.96 & 1.04 & \\
\hline $\begin{array}{l}\text { Donar sangre cuando escucha en la radio o televisión que alguna persona necesita del mismo tipo } \\
\text { de sangre que usted tiene. }\end{array}$ & 0 & 3 & 0.58 & 0.91 & \\
\hline FRUGALIDAD & 0 & 4 & 2.37 & 0.54 & 0.77 \\
\hline Utilizo la misma ropa que la temporada pasada, aunque pueda comprarme nueva. & 0 & 4 & 3.05 & 1.05 & \\
\hline Si mi carro funciona aún bien, no compro uno más nuevo, aun teniendo el dinero & 0 & 4 & 2.98 & 1.38 & \\
\hline Aun teniendo dinero no lo empleo para comprar joyas. & 0 & 4 & 2.95 & 1.4 & \\
\hline Casi siempre como en mi casa, en lugar de ir a restaurantes o taquerías. & 0 & 4 & 2.82 & 1.2 & \\
\hline Vivo sin lujos, aunque pueda dármelos. & 0 & 4 & 2.76 & 1.23 & \\
\hline Si voy a un lugar que no está lejos, prefiero caminar que mover mi carro. & 0 & 4 & 2.74 & 1.45 & \\
\hline Reuso los cuadernos y las hojas de papel que sobran al terminar cada ciclo escolar & 0 & 4 & 2.51 & 1.43 & \\
\hline Una gran parte de mi dinero lo empleo para comprar ropa. & 0 & 4 & 1.47 & 1.38 & \\
\hline Me compro muchos zapatos para que combinen con toda mi ropa. & 0 & 4 & 1.22 & 1.4 & \\
\hline Compro más comida de la que nos hace falta a mí y a mi familia. & 0 & 4 & 1.21 & 1.27 & \\
\hline EQUIDAD & 1.14 & 4 & 3.5 & 0.55 & 0.71 \\
\hline $\begin{array}{l}\text { En mi familia, las niñas tienen la misma oportunidad de estudiar (hasta donde quieran) que los } \\
\text { niños }\end{array}$ & 0 & 4 & 3.84 & 0.58 & \\
\hline Trato a todos mis compañeros como mis iguales, sin importar su origen social & 0 & 4 & 3.74 & 0.67 & \\
\hline Trato a los indígenas de la misma manera que a las personas que no lo son & 0 & 4 & 3.7 & 0.74 & \\
\hline Mi pareja (o novio/novia) tiene el mismo derecho que yo al tomar decisiones sobre cualquier cosa & 0 & 4 & 3.63 & 0.85 & \\
\hline Mi trato para las personas pobres es igual que el que tengo con los más ricos & 0 & 4 & 3.61 & 0.8 & \\
\hline En mi familia, hombres y mujeres tienen las mismas obligaciones en el aseo de la casa & 0 & 4 & 3.33 & 1.09 & \\
\hline $\begin{array}{l}\text { En mi casa, los niños tienen el mismo derecho que los adultos a tomar decisiones importantes } \\
\text { para la familia }\end{array}$ & 0 & 4 & 2.7 & 1.35 & \\
\hline
\end{tabular}


Pág 10

\begin{tabular}{|c|c|c|c|c|c|}
\hline Escalas / Ítems & Min & Max & M & $D E$ & Alfa \\
\hline AUTOCUIDADO & 0.78 & 3.78 & 2.42 & 0.62 & 0.86 \\
\hline Hago cosas que me agradan. & 1 & 4 & 3.11 & 0.9 & \\
\hline Hago cosas que me producen placer. & 0 & 4 & 2.89 & 0.94 & \\
\hline A diario procuro estar bien conmigo mismo(a). & 0 & 4 & 2.89 & 0.95 & \\
\hline Cuando percibo que estoy estresado (a) procuro calmar mi mente & 0 & 4 & 2.69 & 1.1 & \\
\hline A diario enfatizo más en actividades que utilizan la mente que las actividades físicas & 0 & 4 & 2.6 & 1.16 & \\
\hline Hago actividades que me acarrean bienestar. & 0 & 4 & 2.59 & 1.02 & \\
\hline Después de trabajo (o estudio) procuro recompensarme con cualquier actividad placentera. & 0 & 4 & 2.55 & 1.19 & \\
\hline Procuro alimentarme con comidas que le hagan bien a mi salud. & 0 & 4 & 2.53 & 1.04 & \\
\hline Procuro conocerme mejor cada día. & 0 & 4 & 2.45 & 1.16 & \\
\hline Procuro mantener el equilibrio entre mi cuerpo, mis emociones y mi mente. & 0 & 4 & 2.43 & 1.14 & \\
\hline Acostumbro alternar la posición de mi cuerpo a lo largo del día para preservar mi salud. & 0 & 4 & 2.43 & 1.22 & \\
\hline Le presto más atención a mi mente que a mis emociones & 0 & 4 & 2.36 & 1.03 & \\
\hline Participo en actividades que me ayudan a ser una persona mejor. & 0 & 4 & 2.33 & 1.02 & \\
\hline Hago actividades para descansar mi mente de las preocupaciones diarias. & 0 & 4 & 2.14 & 1.29 & \\
\hline Participo en actividades que promueven mi espiritualidad & 0 & 4 & 2.05 & 1.22 & \\
\hline Hago actividad física regularmente para mantener mi salud. & 0 & 4 & 2.01 & 1.23 & \\
\hline Me pongo en estado de meditación para mantener mi mente relajada y alerta. & 0 & 4 & 1.82 & 1.3 & \\
\hline Participo en actividades que me ayudan a conocerme mejor. & 0 & 4 & 1.81 & 1.15 & \\
\hline FELICIDAD & 1.33 & 7 & 5.34 & 1.26 & 0.87 \\
\hline $\begin{array}{l}\text { Algunas personas disfrutan de la vida a pesar de lo que pase, ¿qué tanto crees que esto te } \\
\text { describe a ti? }\end{array}$ & 1 & 7 & 5.36 & 1.48 & \\
\hline Generalmente me considero & 1 & 7 & 5.36 & 1.32 & \\
\hline Comparado(a) con la mayoría de mis conocidos, yo me considero & 1 & 7 & 5.32 & 1.43 & \\
\hline
\end{tabular}

La mayoría de las variables presentaron una interrelación positiva y significativa. Se destacan las relaciones de la orientación al Futuro con las conductas de Autocuidado $(r=0.39 p<0.01)$, Altruismo $(r=0.36 p<0.01)$, el Futuro Trascendental $(r=0.37 p$ $<0.01)$ y Comportamiento Proecológico $(r=0.32 p<0.01)$. En la Tabla 3 se presenta la matriz de correlaciones entre las variables estudiadas.

Tabla 2. Correlaciones entre variables

\begin{tabular}{lcccccccc}
\hline & FUT & FTRA & CPE & ALT & FRU & EQU & AUC & FEL \\
\hline Futuro & 1 & & & & & & & \\
\hline Futuro Trascendental & $.37^{* *}$ & 1 & & & & & & \\
\hline Conducta Proecológica & $.32^{* *}$ & $.24^{* *}$ & 1 & & & & & \\
\hline Altruismo & $.36^{* *}$ & $.26^{* *}$ & $.44^{* *}$ & 1 & & & & \\
\hline Frugalidad & .03 & .009 & $.14^{* *}$ & .09 & 1 & & & \\
\hline Equidad & $.28^{* *}$ & $.20^{* *}$ & $.20^{* *}$ & $.30^{* *}$ & $.26^{* *}$ & 1 & & \\
\hline Autocuidado & $.39^{* *}$ & $.35^{* *}$ & $.37^{* *}$ & $.39^{* *}$ & .08 & $.36^{* *}$ & 1 & \\
\hline Felicidad & .11 & $.17^{*}$ & $.19^{* *}$ & $.22^{* *}$ & .04 & .14 & $.39^{* *}$ & 1 \\
\hline
\end{tabular}

$\mathrm{p}<.05^{*}, \mathrm{p}<.001^{* *}$

FUT =Futuro, FUTRA=Futuro trascendental, $\mathrm{CPE}=$ Comportamiento proecológico, $\mathrm{ALT}=$ Altruismo, FRU=Frugalidad, EQU=Equidad $A U C=$ Autocuidado, $F E L=F e l i c i d a d$ 
Según el modelo de ecuaciones estructurales, las perspectivas temporales de orientación al Futuro (coeficiente estructural=.44) y el Futuro Trascendental (.29), influyen en las Conductas Sustentables, a la vez que estas últimas afectan positivamente a la Felicidad (.38) y a las conductas de Autocuidado (.77). Aunque el modelo no reporta bondad de ajuste estadística $\left(X^{2}=478.52 ; 290\right.$ g.l.; $\left.p<.001\right)$, sí presenta bondad de ajuste práctica (BBNNFI=.91, $C F I=.92 ; R M S E A=.05)$ por lo que se puede concluir su validez.

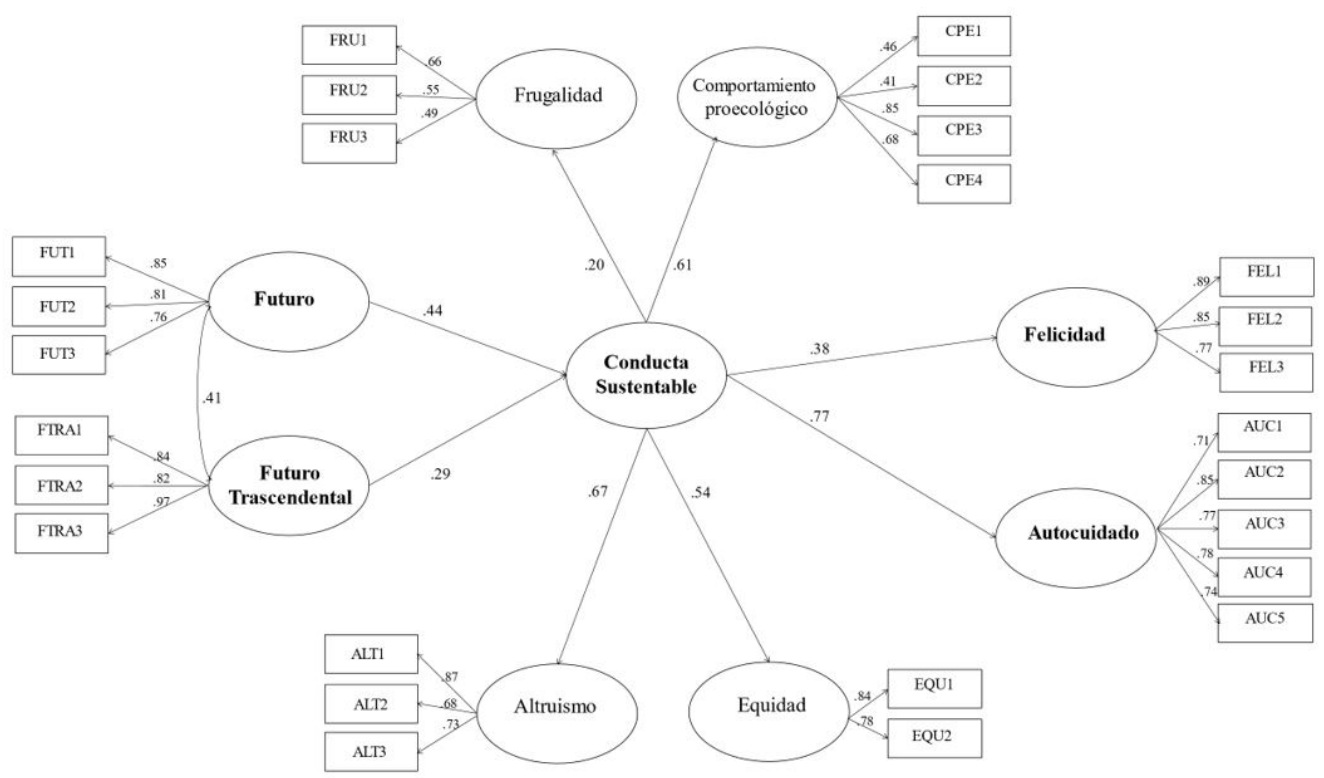

Figura 1. Modelo estructural de conducta sustentable, felicidad y autocuidado, predichas por la orientación al Futuro y al Futuro trascendental. Bondad de ajuste: $X^{2}=478.52$ (290 g.l.) $p=.000, B B N N F I=.91, C F I=.92 ; R M S E A=.05 ; C S R^{2}=.39 ;$ Fel $R^{2}=.14 ; A U C R^{2}=.60$

\section{Discusión}

La presente investigación se enmarcó en la línea de estudios sobre la perspectiva temporal como determinante de las conducta sustentables. Esta línea plantea la idea de que la orientación al futuro constituye un factor importante en la determinación a actuar de manera prosocial y proambiental. Estudios previos apoyan dicha idea (Fraijo et al., 2012; Milfont \& Gouveia, 2006), sin embargo, ninguno de ellos se había abocado a investigar la relación de perspectiva del futuro trascendental con las CS, por lo que un propósito especial del presente estudio fue llenar ese vacío.

Los resultados del modelo probado apoyan la hipótesis de que la orientación al futuro trascendental funciona como predictor de las CS, tal como lo hace la orientación al futuro en su acepción tradicional. Los resultados adicionales del modelo ratificaron hallazgos previos en el sentido de que las conductas protectoras del medio ambiente físico y social generan felicidad (Barrera-Hernández et al., 2020; Corral-Verdugo et al., 2011), y que las conductas de autocuidado se relacionan con las CS (Pato \& Corral, 2013).

Coinciden también con lo encontrado en investigaciones anteriores respecto a la influencia de la perspectiva temporal futura de las personas en las prácticas de conductas a favor del ambiente físico y social (Corral-Verdugo et al., 2006; Enzler, 2015), 
Pág 12

El presente estudio confirmó el hallazgo de que las conductas de autocuidado se asocian a las conductas proecológicas, frugales, altruistas y equitativas, como lo habían reportado previamente Pato y Corral (2013); y además encontró que el autocuidado se relaciona significativamente con las dos orientaciones temporales del futuro y con el reporte de felicidad. así como con estudios previos acerca de las relaciones entre las CS y la felicidad (Brown \& Kasser, 2005; Corral-Verdugo et al., 2011; Choi, 2016). Todos estos resultados parecen reafirmar la convicción de que la orientación de las personas hacia la sostenibilidad se fundamenta en la anticipación, la planeación y la preocupación por el bienestar de otros en el futuro, así como en la necesidad de garantizar la calidad ambiental en tiempos venideros.

Aparte de lo anterior, los hallazgos más destacados del estudio que aquí se reporta incluyen el de la correlación positiva y significativa entre las perspectivas temporales de futuro y futuro trascendental, así como entre estas dos con las CS; además de la confirmación de que existe una relación entre esas conductas y los comportamientos de autocuidado. Estos hallazgos implican, en primer lugar, que los individuos que se orientan al futuro más allá de su vida (basados en sus convicciones religiosas o espirituales) tienden también a preocuparse por lo que ocurrirá en el futuro "terrenal". Es verdad que las convicciones religiosas, especialmente las fatalistas ("todo está bajo el control de Dios y nosotros no podemos hacer nada") y las escatológicas ("el mundo acabará pronto") pueden llevar a las personas a despreocuparse por el futuro del planeta y de otras personas (ver Sachdeva, 2016, por ejemplo); pero si esas convicciones no son extremistas y tienen un origen espiritual, pueden lograr que el futuro trascendental se asocie a un futuro más tangible en este mundo, como parecen mostrarlo los principales resultados. En segundo lugar, el hecho de que la orientación al futuro trascendental no sólo se asoció a la propensión al futuro, sino también a las CS, revelaría que la preocupación por alcanzar un tiempo que trasciende la vida se apoya, entre otras cosas, en un interés por la integridad ambiental aquí y ahora. Este hallazgo se relaciona con otros que muestran que las creencias religiosas llevan a aceptar la obligación moral de cuidar la naturaleza (Baylor \& Brandhorst, 2015) y a practicar acciones de cuidado ambiental (Tarakeshwar, Swank, Pargament, \& Mahoney, 2001). Lo novedoso aquí, es que esas creencias religiosas se manifiestan como futuro trascendental.

El presente estudio confirmó el hallazgo de que las conductas de autocuidado se asocian a las conductas proecológicas, frugales, altruistas y equitativas, como lo habían reportado previamente Pato y Corral (2013); y además encontró que el autocuidado se relaciona significativamente con las dos orientaciones temporales del futuro y con el reporte de felicidad. De acuerdo con estos resultados, se puede entonces suponer que las personas que poseen una mayor orientación al futuro y al futuro trascendental practicarán más conductas sustentables y experimentarán más felicidad y conductas de autocuidado. También se podrían interpretar en el sentido de que las personas que cuidan de sí mismos tienden a cuidar de otras personas y del ambiente, a la vez que se consideran más felices y mantienen conductas en el presente con objetivos en el futuro dentro y más allá de su vida.

Entre algunas limitaciones del estudio se encuentra, por un lado, su naturaleza correlacional, la cual presenta desventaja frente a estudios experimentales o longitudinales que permiten realizar seguimiento al comportamiento de las variables en el tiempo. Además, los instrumentos de medición utilizados consistieron en autoreportes de los participantes respecto a su comportamiento y creencias, siendo posible que los autoreportes conductuales no sean necesariamente equivalentes a las conductas actuales. Por otro lado, el amplio rango de edad de los participantes limita la representatividad de los resultados en grupos de edad específicos, no obstante, el objetivo de la investigación se orientó a la población general. Pese a estas limitaciones, los datos encontrados en esta investigación aportan información potencialmente valiosa 
que ayuda a elucidar las características que posee un individuo que orienta su conducta a los ideales de la sostenibilidad. Una de esas características, la orientación al futuro trascendental posee un potencial que aún no se ha explorado ni incorporado en estrategias de promoción de las CS que incluyen el componente religioso. En estudios posteriores, que consideren las limitaciones aquí encontradas, se podría avanzar aún más en la construcción de un esquema de relaciones entre las conductas sustentables, la orientación temporal -en especial la del futuro trascendental- y las consecuencias psicológicas positivas que se desprenden de las prácticas proambientales.

\section{Referencias}

Arnocky, S., Milfont, T., \& J. Nicol. (2014). Time perspective and sustainable behavior: Evidence for the distinction between consideration of immediate and future consequences. Environment and Behavior, 46, 556-582. http://doi. org/10.1177/0013916512474987

Barrera-Hernández, L. F., Corral-Verdugo, V., Fraijo-Sing, B. S., \& Tapia-Fonllem, C. 0. (2018). Psychological and physical indicators of a transcendental positive environment and its impact on sustainable behavior. Journal of Psychological \& Educational Research, 26(1) 51-68.

Barrera-Hernández, L. F., Sotelo-Castillo, M. A., Echeverría-Castro, S. B., \& Tapia-Fonllem, C. O. (2020). Connectedness to Nature: Its Impact on Sustainable Behaviors and Happiness in Children. Frontiers in Psychology, 11, 276. http://doi.org/10.3389/ fpsyg. 2020.00276

Baylor, R. J., \& Brandhorst, S. R. (2015). Religiosity and environmental attitudes: Engagement in proenvironmental behavior. Psi Chi Journal of Psychological Research, 20, 58-64. http://doi.org/10.24839/2164-8204.jn20.2.58

Boniwell, I., \& Zimbardo, P.G. (2004). Balancing One's Time Perspective in Pursuit of Optimal Functioning. En P. A., Linley, \& S., Joseph. (Eds.), Positive psychology in practice. Hoboken, $\mathrm{NJ}$ : Wiley.

Boyd, J. N., \& Zimbardo, P. G. (1997). Constructing time after death. The transcendental-future time perspective. Time \& Society, 6, 35-54. http://doi. org/10.1177/0961463X97006001002

Brown, K. W., \& Kasser, T. (2005). Are psychological and ecological well-being compatible? The role of values, mindfulness, and lifestyle. Social Indicators Research, 74, 349-368. http://doi.org/10.1007/s11205-004-8207-8

Choi, J. (2016). Sustainable Behavior: Study Engagement and Happiness among University Students in South Korea. Sustainability, 8, 599. http://doi.org/10.3390/ su8070599

Corral, V., \& Pinheiro, J. (2004). Aproximaciones al estudio de la conducta sustentable. Medio Ambiente y Comportamiento Humano, 5, 1-26.

Corral, V. (2010). Psicología de la sustentabilidad. Un análisis de lo que nos hace pro ecológicos y prosociales. México: Trillas

Corral, V. (2012). Sustentabilidad y Psicología Positiva: Una visión optimista de las conductas proambientales y prosociales. México: El Manual Moderno.

Corral, V., Frías, M., Gaxiola, J., Fraijo, B., Tapia, C., \& Corral, N. (2014). Ambientes positivos: Ideando entornos sostenibles para el bienestar humano y la calidad ambiental. México: Pearson

Corral-Verdugo, V., Fraijo-Sing, B., \& Pinheiro, J. Q. (2006). Sustainable behavior and time perspective: Present, past, and future orientations and their relationship with water conservation behavior. Interamerican Journal of Psychology, 40, 139-147.

Corral-Verdugo, V., García, C., Castro, L., Viramontes, I., \& Limones, R. (2010). Equity and sustainable lifestyles. En V. Corral-Verdugo, C. García, \& M. Frías (Eds.), Psychological Approaches to Sustainability. New York: Nova Science Publishers. 
Corral-Verdugo, V., Mireles-Acosta, J., Tapia-Fonllem, C., \& Fraijo-Sing, B. (2011). Happiness as correlate of sustainable behavior: A study of pro-ecological, frugal, equitable and altruistic actions that promote subjective wellbeing. Human Ecology Review, 18, 95-104.

Csutora, M., \& Zsóka, Á. (2012). Relation of spirituality to happiness, life satisfaction and sustainable lifestyles. Recuperado de: http://core.ac.uk/download/ files/481/12355453.pdf

Enzler, H. B. (2015). Consideration of future consequences as a predictor of environmentally responsible behavior evidence from a general population study. Environment and Behavior, 47, 618-643. http://doi.org/10.1177/0013916513512204

Fraijo, B., Corral, V., Tapia, C., \& García, F. (2012). Adaptación y prueba de una escala de orientación hacia la sustentabilidad en niños de sexto año de educación básica. Revista Mexicana de Investigación Educativa, 17, 1091-1117.

Kerlinger, F., \& Lee, H. (2002). Investigación del comportamiento. Métodos de investigación en ciencias sociales. México: McGraw Hill.

Landes, X., Unger, C., Andsbjerg, K., Frank, K., \& Wiking, M. (2015). Sustainable happiness: Why waste prevention may lead to an increase in quality of life. Kbh: Danish Ministry of the Environment. http://mst.dk/media/130530/141203-sustainable-happiness.pdf

Lee, R. L. (2009). The re-enchantment of time. Death and alternative temporality. Time \& Society, 18, 387-408. http://doi.org/10.1177/0961463X08099951

Lyubomirsky, S., \& Lepper, H. (1999). A measure of subjective happiness: Preliminary reliability and construct validation. Social Indicators Research, 46, 137155. http://doi.org/10.1177/0961463X08099951

Madjar, M., \& Ozawa, T. (2006). Happiness and sustainable consumption: psychological and physical rebound effects at work in a tool for sustainable design. The International Journal of Life Cycle Assessment, 11, 105-115.

Manríquez-Betanzos, J. C., Corral-Verdugo, V., Vanegas-Rico, M. C., Fraijo-Sing, B. S., \& Tapia-Fonllem, C. O. (2016). Positive (gratitude, eudaimonia) and negative (scarcity, costs) determinants of water conservation behaviour. Psyecology, 7, 178200. http://doi.org/10.1080/21711976.2016.1149986

Milfont, T. L., \& Gouveia, V. V. (2006). Time perspective and values: An exploratory study of their relations to environmental attitudes. Journal of Environmental Psychology, 26, 72-82. http://doi.org/10.1016/i.jenvp.2006.03.001

Ortuño, V. E., Paixão, M. P., \& Nunes, I. J. (2011). Diferenças na perspectiva temporal entre estudantes religiosos e não religiosos. Actas do VIII Congresso Iberoamericano de Avaliacão Psicologica. Lisboa: Universidade de Lisboa.

Ortuño, V. E., Paixão, M. P., \& Nunes, I. J. (2013). Tempus Post mortem?: Adaptação portuguesa da transcendental-future time perspective scale (TFTPS). Avances en Psicología Latinoamericana, 31, 376-388.

Pato, C., \& Corral, V. (2013, julio). Cuidado consigo mesmo e cuidado del ambiente. Trabajo presentado en el XXXIV Congreso Interamericano de Psicología. Brasilia, Brasil.

Sachdeva, S. (2016). Religious identity, beliefs, and views about climate change. In Oxford Research Encyclopaedia. Oxford, UK: Oxford University Press.

Seema, R., Baltin, A., \& Sircova, A. (2014). Transcendental future-is it a healthy belief or a time perspective? The transcendental-future time perspective inventory (TTPI) in Estonian. Trames, 1, 57-75. http://doi.org/10.3176/tr.2014.1.04

Tapia, C., Fraijo, B., Corral, V., Gutiérrez, C., \& Tirado, H. (2006). Validación de una escala de orientación hacia la sustentabilidad. En B. Fraijo, S. Echeverría, \& C. Tapia. (Eds.), Desierto y Mar. Estudios Sociales en Sonora. Cd. Obregón, México: Instituto Tecnológico de Sonora. 
Tapia-Fonllem, C., Corral-Verdugo, V., Fraijo-Sing, B., \& Durón-Ramos, M. F. (2013). Assessing sustainable behavior and its correlates: A measure of pro-ecological, frugal, altruistic and equitable actions. Sustainability, 5(2), 711-723. http://doi. org/10.3390/su5020711

Tarakeshwar, N., Swank, A. B., Pargament, K. I., \& Mahoney, A. (2001). The sanctification of nature and theological conservatism: A study of opposing religious correlates of Environmentalism. Review of Religious Research, 42, 387- 404. http://doi. org/10.2307/3512131

Tobón, O. C. (2003). El autocuidado una habilidad para vivir. Hacia Promoción de la Salud, 8, 37-49.

Van Beek, W., \& Kairys, A. (2015). Time Perspective and Transcendental Future Thinking. In M. Stolarski, N. Fieulaine, \& W. van Beek (Eds.), Time Perspective Theory; Review, Research and Application (pp. 73-86). Springer International Publishing.

Zimbardo, P., \& Boyd, J. (1999). Putting Time in Perspective: A Valid, Reliable individual differences Metric. Journal of Personality and Social Psychology, 77(6), 1271-1288. http://dx.doi.org/10.1037/0022-3514.77.6.1271 